\title{
Simultaneous Enantiospecific Detection of Multiple Compounds in Mixtures using NMR Spectroscopy
}

\author{
Lars T. Kuhn, ${ }^{[a]}$ Kumar Motiram-Corral, ${ }^{[b]}$ Toby J. Athersuch, ${ }^{[c]}$ Teodor Parella, ${ }^{[b]}$ and Míriam Pérez- \\ Trujillo*[b] \\ [a] Dr. L. T. Kuhn \\ Institut für Physikalische Chemie \\ Albert-Ludwigs-Universität Freiburg \\ Albertstraße 21 \\ D-79104 Freiburg \\ Germany \\ [b] K. Motiram-Corral, Dr. T. Parella, Dr. M. Pérez-Trujillo \\ Servei de Ressonància Magnètica Nuclear \\ Facultat de Ciències i Biociències \\ Universitat Autònoma de Barcelona \\ E-08193 Cerdanyola del Vallès \\ Catalonia \\ Spain \\ E-mail: miriam.perez@uab.cat \\ [c] Dr. T. J. Athersuch \\ Division of Systems Medicine \\ Department of Metabolism, Digestion and Reproduction \\ Imperial College London \\ South Kensington \\ London \\ UK \\ Supporting information for this article is given via a link at the end of the document
}

Abstract: Chirality plays a fundamental role in nature, but its detection and quantification still face many limitations. To date, the enantiospecific analysis of mixtures necessarily requires prior separation of the individual components. The simultaneous enantiospecific detection of multiple chiral molecules in a mixture represents a major challenge, which would lead to a significantly better understanding of the underlying biological processes; e.g. via enantiospecifically analysing metabolites in their native environment. Here, we report on the first in situ enantiospecific detection of a thirty-nine-component mixture. As a proof of concept, eighteen essential amino acids at physiological concentrations were simultaneously enantiospecifically detected using NMR spectroscopy and a chiral solvating agent. This work represents a first step towards the simultaneous multicomponent enantiospecific analysis of complex mixtures, a capability that will have substantial impact on metabolism studies, metabolic phenotyping, chemical reaction monitoring, and many other fields where complex mixtures containing chiral molecules require efficient characterisation.

Chirality is a property fundamental to biological processes at a molecular level. Nevertheless, challenging questions regarding chiral analysis remain open and need to be addressed. ${ }^{[1]}$ Many mirror-image (enantiomeric) molecular pairs occur naturally, each of them having different functionalities, biological activities, and sometimes origin, such as in the case of amino acids (AAs). D-amino acids (AAs) - though in smaller quantities than L-AAs are widely distributed in living organisms and their study has led to significant advances in the fields of microbiology and medicine, among others. For instance, D-serine and D-aspartic acid act as neurotransmitters in the human brain, ${ }^{[2]}$ abnormal DAA levels have been associated to various diseases including schizophrenia ${ }^{[3]}$ or $\mathrm{ALS}^{[4]}$ and D-AAs play a relevant role in bacterial metabolism ${ }^{[5]}$ and in the host-microbe interface. ${ }^{[6]}$ Hence, the differentiation and identification of enantiomeric metabolites is of paramount importance to biological applications. Enantiospecific analyses are crucial in metabolism studies, as well as in metabolic profiling, and are mandatory in drug research. ${ }^{[7]}$ Despite of this fact, many of these investigations rely on non-enantiospecific analytical methods, which overlook the stereochemistry of the metabolites involved.

Enantiospecific detection can be achieved using appropriate spectroscopic techniques ${ }^{[8-10]}$ and via methods based on asymmetric separation platforms. ${ }^{[1]}$ However, these methods necessarily require the isolation of each enantiomeric pair or each individual enantiomer from its original matrix before detection (e.g. metabolites in a body fluid, in a cell or in a plant extract). Separation steps potentially introduce additional challenges and significant risks to the analysis, e.g. sample degradation, stereochemical instability of analytes, sample contamination or analyte loss, and involve time- and costconsuming procedures.

The direct and simultaneous enantiospecific detection of multiple compounds in a complex mixture would give access to a new level of complexity (i.e. enantiospecific data) from metabolites in their natural media and conditions, providing more precise information to explain better the biological processes under observation. Developing robust and efficient methods for such analyses represents a major analytical challenge, potentially explaining the limited number of studies demonstrating simultaneous multicomponent enantiospecific detection reported in the literature. In these examples, mixtures 
of two or three enantiomeric pairs were enantiodifferentiated using extremely non-physiological conditions utilizing methodologies whose applicability to more complex mixtures seems difficult. ${ }^{[12-14]}$ In a previous study, we enantiodifferentiated racemic RS-ibuprofen within a complex mixture (human urine) using NMR spectroscopy in combination with a chiral solvating agent (CSA) while introducing the concept of chiral metabolomics. ${ }^{[15]}$ The CSA-NMR method is based on the formation of diastereomeric complexes between analytes and the chiral agent, resulting in chemical shift differences between enantiomers (enantiodifferentiation, $\Delta \Delta \delta$ ) observed in the NMR spectra. ${ }^{[16-18]}$

Here, we show for the first time the simultaneous enantiospecific detection of eighteen chiral metabolites within an aqueous mixture at physiological concentrations. A mixture corresponding to the twenty essential amino acids (AAs) in a low millimolar concentration range was analysed. The sample contained the nineteen chiral essential AAs present as racemates plus glycine, giving a thirty-nine-component mixture, mixture 1 (Table $\mathrm{S}-1$ ). Out of the twenty spectroscopically detected amino acids, fifteen were simultaneously enantiodifferentiated conducting a rapid ${ }^{1} \mathrm{H}$ NMR experiment only following the addition of a small amount of the CSA (-)-(18crown-6)-2,3,11,12-tetracarboxylic acid, (-)-18C6 $\mathrm{H}_{4}$. In total, eighteen out of the nineteen chiral AAs were enantiospecifically detected using a combination of ${ }^{1} \mathrm{H}$ and ${ }^{13} \mathrm{C}$ NMR spectroscopy. $(-)-18 \mathrm{C}^{2} \mathrm{H}_{4}$ is a water soluble $\mathrm{CSA}$, which has proven to enantiodiscriminate isolated enantiomeric pairs of a wide variety of molecules such as alpha- and beta-AAs, amino alcohols or alkyl amines, among others. ${ }^{[19,20]}$
Initially, mixture 1 was titrated with (-)- $18 \mathrm{C} 6 \mathrm{H}_{4}$ and monitored via the acquisition of a standard $1 \mathrm{D}{ }^{1} \mathrm{H}$ NMR spectrum. After subsequent additions of the CSA, we observed that a relatively small amount (3.1 $\mathrm{mg}$, corresponding to a final total concentration of $11.7 \mathrm{mM}$ ) induced enantiospecific changes in the spectrum that were sufficient to enantiodifferentiate most of the enantiomeric pairs of $A A s$ in the mixture. Figure $1 \mathrm{a}-\mathrm{c}$ shows the full $1 \mathrm{D}{ }^{1} \mathrm{H}$ and $1 \mathrm{D}^{13} \mathrm{C}$ NMR spectra of mixture 1 as well as a few highlighted expanded regions of the spectra acquired before and after the addition of the chiral agent (Figure $1 \mathrm{~b}$ and Figure 1c, respectively). The analysis of a similar AA mixture, enriched with L-enantiomers, mixture 2 (Table $\mathrm{S}-1$ ), allowed the enantiodifferentiated spectral features to be matched unambiguously to their corresponding $\mathrm{D}$ - or L-enantiomer (Figure 1d). The method described above enabled the direct enantiospecific observation of the original mixture and provided access to stereochemical information about enantiomeric components via a simple spectral output, resulting in a ${ }^{1} \mathrm{H}$ and a ${ }^{13} \mathrm{C}$ NMR-based enantiospecific molecular profile of mixture 1 (Figure S-1 and Figure S-2).

In a second step, we conducted more advance NMR correlation experiments on mixture 1 after CSA addition (Figure 2). The ${ }^{1} \mathrm{H}$ pure shift ${ }^{[21]} \mathrm{J}$-resolved experiment, PS-JRES, allowed proton signals corresponding to resolved enantiomeric pairs to be clearly distinguished. These pairs were observed as identical, duplicated signals, displaced in the direct dimension by some ppb corresponding to $\Delta \Delta \delta\left({ }^{1} \mathrm{H}\right)$ (Figure 2a). A skyline projection in the direct dimension yielded a pure shift $1 \mathrm{D}{ }^{1} \mathrm{H}$ NMR spectrum representing an enantiospecific molecular profile of the mixture (Figure S-1). Importantly, this profile exhibited significantly less signal overlap and higher spectral resolution
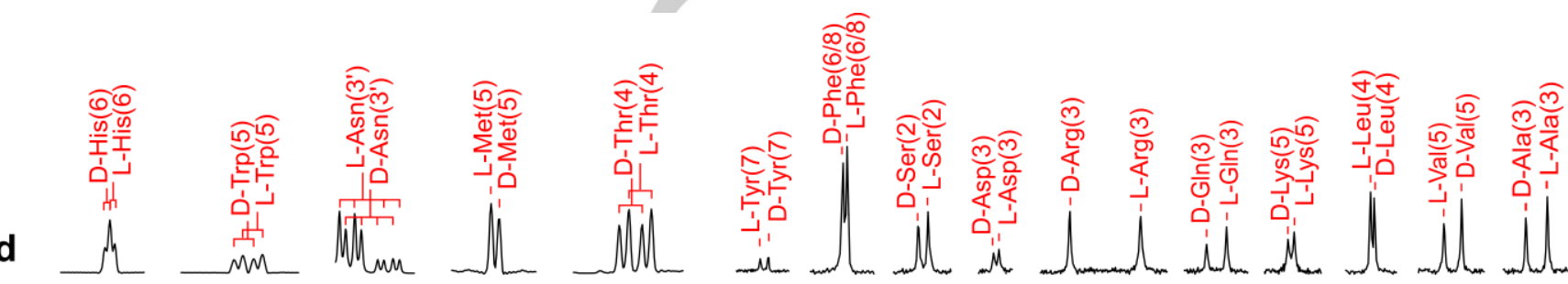

C
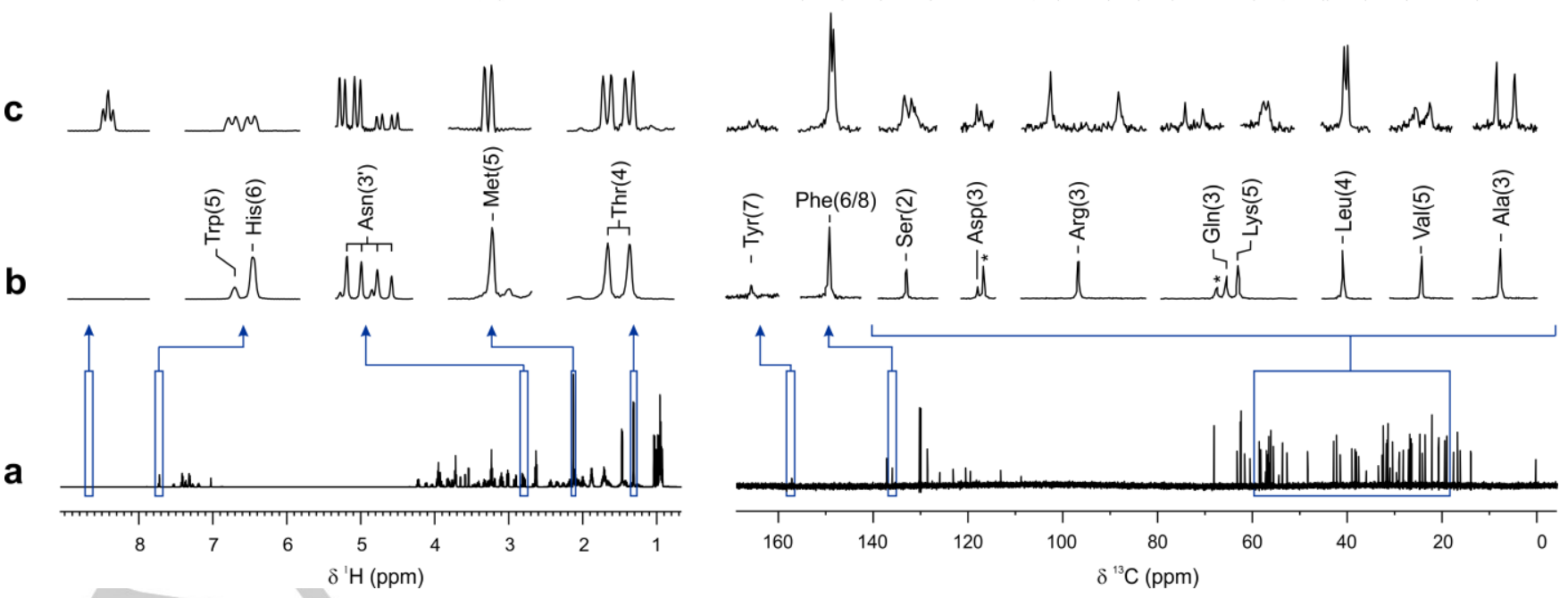

Figure 1. Simultaneous enantiospecific detection of a AA mixture by NMR spectroscopy. (a) $1 \mathrm{D}{ }^{1} \mathrm{H}$ and ${ }^{13} \mathrm{C}$ NMR spectra of mixture 1. (b) Highlighted ${ }^{1} \mathrm{H}$ and ${ }^{13} \mathrm{C}$ NMR signals of mixture 1. (c) Same NMR signals after the addition of $3.1 \mathrm{mg}$ (final concentration $11.7 \mathrm{mM}$ ) CSA (-)-18C6H4. (d) Analogous to d but corresponding to mixture 2, enriched with L-enantiomers. The enantiospecific assignment of spectral signals to the corresponding D/L-AA is indicated (number in brackets refers to the specific AA nucleus, see Table S-2). 


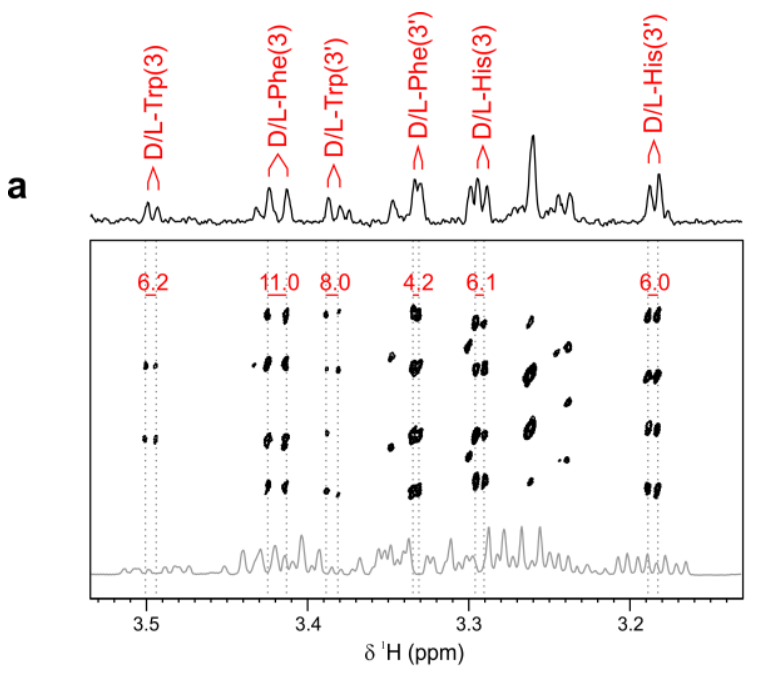

b

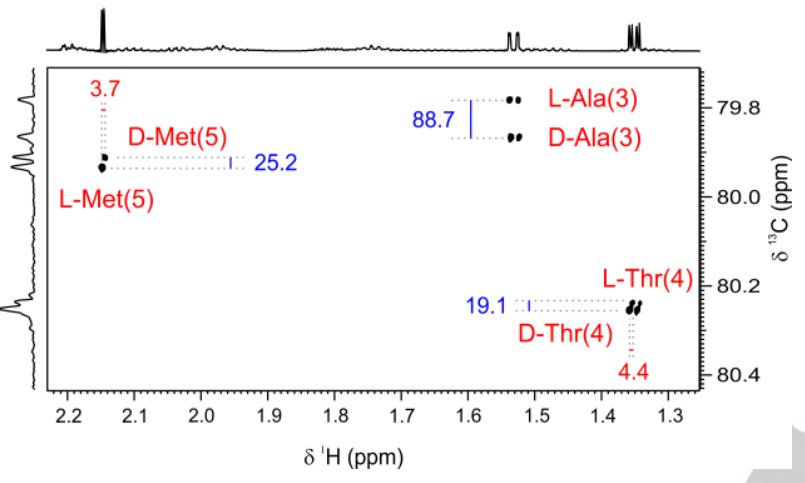

Figure 2. Example of state-of-the-art NMR experiments applied to mixture 1 (a) Extension of PS-JRES spectrum, showing the internal projection (top) and a standard 1D ${ }^{1} \mathrm{H}$ NMR experiment (bottom). (b) Extension of SA-HSQC correlation, showing the ${ }^{13} \mathrm{C} \quad \mathrm{NMR}$ internal projection (left). Enantiodifferentiated AAs NMR signals and their values of $\Delta \Delta \delta\left({ }^{1} \mathrm{H}\right)$ (red) and $\Delta \Delta \delta\left({ }^{13} \mathrm{C}\right)$ (blue), in $\mathrm{ppb}$, are indicated.

than the standard $1 \mathrm{D}{ }^{1} \mathrm{H}$ NMR spectrum due to the elimination of signal $J_{H H}$ multiplicities obtained using the pure shift scheme (Figure 2a, top, vs Figure 2a, bottom). On the other hand, a spectrally aliased (SA) ${ }^{1} \mathrm{H},{ }^{13} \mathrm{C}$ - HSQC experiment enabled the simultaneous enantiospecific detection of ${ }^{1} \mathrm{H}$ and ${ }^{13} \mathrm{C}$ nuclei and the measurement of $\Delta \Delta \delta\left({ }^{1} \mathrm{H}\right)$ and $\Delta \Delta \delta\left({ }^{13} \mathrm{C}\right)$ of enantiodifferentiated signals, due to a significant increase in the resolution observed in the ${ }^{13} \mathrm{C}$ dimension compared to a conventional ${ }^{1} \mathrm{H},{ }^{13} \mathrm{C}$ - HSQC (Figure 2b). ${ }^{[22,23]}$ For the identification of the individual components of mixture 1 before the addition of the CSA, an NMR spectral data base commonly used in metabolomics research was utilized to assign ${ }^{1} \mathrm{H}$ and ${ }^{13} \mathrm{C}$ signals. ${ }^{[24]}$ After addition of the CSA, some signals of the original spectrum were split into two (enantioresolved) and most of the signals were slightly shifted with respect to the original spectrum. In this case, the spectral assignment workflow incorporated the NMR experiments described above with standard ${ }^{1} \mathrm{H},{ }^{1} \mathrm{H}-\mathrm{TOCSY}$ and ${ }^{1} \mathrm{H},{ }^{13} \mathrm{C}-\mathrm{HSQC}$ correlations (Figure S-3 and Figure S-4).

The enantiospecificity of the experiment was evaluated by the analysis of the enantiodifferentiated NMR signals and by the measurement of the parameters $\Delta \Delta \delta$ (enantiodifferentiation) and $\mathrm{E}$ (enantioresolution quotient, which provides information about the quality of the signal resolution ${ }^{[25]}$ of each split signal. Further, the sign of $\Delta \Delta \delta$ (and $\mathrm{E}$ ) was determined, which is related to the
$\mathrm{D} / \mathrm{L}$ identification; i.e. the determination of the absolute configuration of the enantiodiscriminated AAs. ${ }^{[16,25]}$ The results of the enantiospecific detection of mixture 1 are summarized in Table S-2. In total, eighteen out of nineteen chiral AAs were enantiodiscriminated, all of which showed more than one enantiodifferentiated signal (except DL-Tyr). Fifteen AAs pairs were enantiodiscriminated via ${ }^{1} \mathrm{H}$ NMR spectroscopy and sixteen via ${ }^{13} \mathrm{C}$ NMR spectroscopy, respectively. In thirteen $A A$ pairs, enantiodifferentiation was observed by both nuclei. In the case of ${ }^{1} \mathrm{H}$ - from the PS-JRES internal projection (Figure S-1) - thirtyeight ${ }^{1} \mathrm{H}$ signals were enantiodifferentiated and identified, nineteen of them with $E \geq 1.0$ and only six of them with $E<0.5$; $\Delta \Delta \delta$ and $E$ values varied from 86.0 to $3.0 \mathrm{ppb}$ and from 11.5 to 0.3 , respectively. For ${ }^{13} \mathrm{C}$ - taken from the $1 \mathrm{D}{ }^{13} \mathrm{C} N M R$ experiment (Figure S-2) - fifty NMR signals were enantiodifferentiated and identified, twenty-three of them with $E \geq 1.0$ and only six of them with $E<0.5 ; \Delta \Delta \delta$ and $E$ values varied from 307.2 to $10.0 \mathrm{ppb}$ and from 6.8 to 0.3 , respectively. In addition, all but two enantiodifferentiated AAs pairs were D/L identified, and in most cases more than one split signal was assigned to the corresponding enantiomer. In summary, excellent enantiodiscrimination results were observed by detection of both ${ }^{1} \mathrm{H}$ and ${ }^{13} \mathrm{C}$ nuclei.

To date, the CSA-NMR approach has been used to enantiorecognize just one enantiomeric pair at a time and, except in very few cases, ${ }^{[15,26]}$ with the sample being isolated from its original medium. Interestingly, when enantiospecifically detecting (complex) mixtures using NMR spectroscopy, the relatively low values of $\Delta \Delta \delta$ yielded by CSAs compared to other NMR auxiliaries (e.g. chiral derivatizing agents) turn out to be an advantage, as this facilitates the identification of resolved enantiomeric pairs in the complex spectra. Signals corresponding to an enantiomeric pair are normally close to one another. When applying the described method for the first time on a mixture, it is convenient to start titrating the sample with the CSA while monitoring it by ${ }^{1} \mathrm{H}$ NMR to determine the quantity of CSA needed for a proper enantiodifferentiation according to the specific objective (e.g. targeted or untargeted study). Further, following peak shifts during titration can help identifying signals corresponding to the same enantiomeric pair. Apart from adding more CSA, another way to increase $\Delta \Delta \delta$ and improve signal enantioresolution $(E)$ consists in decreasing the temperature of the NMR experiment. ${ }^{[25]}$ This is helpful, for instance, when there is a good overall $\Delta \Delta \delta$ of signals but a specific target peak is not sufficiently enantioresolved (exemplified for the H-6 signal of DLhistidine in Figure 3). In addition, the absence of chemical reactions between the CSA and sample components avoids kinetic resolution, and, combined with the inherently quantitative nature of NMR spectroscopy, allows for quantitative enantiospecific methods to be considered, e.g. by conducting a quantitative NMR experiment. ${ }^{[27]}$

Finally, an issue that attracted our attention was the relatively small amount of CSA (-)- $18 \mathrm{C}^{2} \mathrm{H}_{4}(3.1 \mathrm{mg}, 11.7 \mathrm{mM})$ needed to yield the described results, i.e. to induce the enantiodifferentiation of eighteen AAs totalling a concentration of $42.3 \mathrm{mM}$. This means that only ca. 0.3 equivalents on average of CSA were required for each enantiodiscriminated $A A$. Typically, $\geq 1$ eq. is required for this type of experiments for an isolated enantiomeric pair. ${ }^{[17-20]}$ To explore this observation further, a similar experiment was performed separately to four 


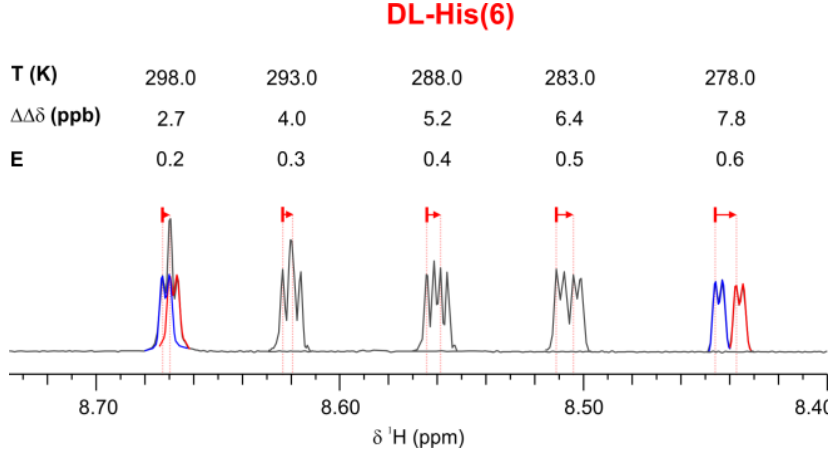

Figure 3. Increase of signal enantioresolution with temperature decrease. DLHis(6) signal region of ${ }^{1} \mathrm{H}$ NMR spectra of mixture 1 after the addition of 3.1 $\mathrm{mg}(11.7 \mathrm{mM})$ of CSA (-)- $18 \mathrm{C} \mathrm{H}_{4}$ at temperatures indicated. D- and L-His(6) signals shown in blue and red, respectively.

enantiomeric pairs randomly selected from those in mixture 1 (DL-alanine, DL-asparagine, DL-methionine and DL-threonine); identical $A A$ and CSA concentrations were used but, this time, dissolved in pure aqueous solutions. In this experiment, the relative proportions of CSA to AA (equivalents of CSA) were higher than in mixture 1 , ranging from 2.7 to 4.8 depending on the AA (the effective CSA/AA ratio would correspond to the experimental ratio shown in Table $S-2$ ). To reach these conditions, the individual titration of each AA with the CSA was carried out. Based on this, we initially hypothesized that the degree of enantiomeric differentiation (i.e. values of $\Delta \Delta \delta$ ) for the pure AAs would be much greater than in the presence of the other AAs on account of the reduced competition for interaction with the CSA. Remarkably, the results obtained for pure AAs were comparable to those obtained within the mixture and did not support this hypothesis (Table S-3). In general, slightly higher values were obtained for pure AAs, with some exceptions like C-4 of DL-threonine, C-2 and C-5 of DL-methionine or C-2 of DL-asparagine, among others. The $\Delta \Delta \delta$ values of pure AAs, corresponding to an addition of 0.3 equivalents of CSA (see titration curves in Figure S-5), and the $\Delta \Delta \delta$ values of same AAs in mixture 1 (Table S-2) were also compared. The results showed much greater $\Delta \Delta \delta$ values, i.e. a better enantiodifferentiation, when the AAs were present in the mixture. Thus, these results suggest that there is a synergistic effect that greatly favours enantiodifferentiation due to the presence of other (chiral) molecules in the solution, i.e. a mixture effect. ${ }^{[28]}$ We also observed that the same signals were enantiodifferentiated (having the same $\Delta \Delta \delta$ sign) as compared to the mixture in practically all cases. This result suggests that similar AA-CSA diastereomeric complexes are formed in pure solution and in the mixture. Additional experiments are currently being carried out to explore this effect in greater detail.

In summary, we have demonstrated the simultaneous enantiospecific detection of multiple chiral metabolites within an aqueous mixture, at physiological concentrations and without a prior separation or derivatization of its components. This is, to the best of our knowledge, without precedent. Eighteen essential AAs were enantiospecifically detected within a thirty-ninecomponent mixture using NMR spectroscopy and a small quantity of a CSA. Furthermore, the method allowed the detection and identification of all components, yielding a ${ }^{1} \mathrm{H}$ and ${ }^{13} \mathrm{C}$ NMR-based enantiospecific molecular profile of the mixture in a straightforward way with only minimal manipulation and a concomitant structural preservation of the sample. Compatible with untargeted and targeted quantitative studies, this work represents a first step towards the direct multicomponent enantiospecific analysis of complex mixtures, showing significant potential for application in metabolism studies, metabolic phenotyping, and in chemical reaction analysis, among others. Future research will include the study of other small-molecule metabolite mixtures and the evaluation of other compatible CSAs.

\section{Acknowledgements}

Financial support provided by the Spanish Ministry of Economy and Competitiveness (project PGC2018-095808-B-100) is gratefully acknowledged. We also thank the "Servei de Ressonància Magnètica Nuclear (SeRMN)" of the Universitat Autònoma de Barcelona (UAB) for the generous allocation of spectrometer time.

Keywords: complex mixture, simultaneous enantiospecific analysis, mixture effect, multicomponent, NMR spectroscopy

[1] D. Kondepudi in Chiral Analysis. Advances in Spectroscopy, Chromatography and Emerging Methods (Ed.: P. L. Polavarapu), Elsevier, 2018, pp. 3-28.

[2] a) F. Hucho, Angew. Chem. Int. Ed., 2000, 39, 2849-2850; b) A Hashimoto, T. Oka, Prog. Neurobiol. 1997, 52, 325-353.

[3] I. Chumakov, M. Blumenfeld, O. Guerassimenko, L. Cavarec, M. Palicio, H. Abderrahim, et al. Proc. Natl. Acad. Sci. U. S. A. 2002, 99, 1367513680.

[4] J. Sasabe, T. Chiba, M. Yamada, K. Okamoto, I. Nishimoto, M. Matsuoka, S. Aiso, Embo J. 2007, 26, 4149-4159.

[5] L. Hubert, O. Dong-Chan, F. Cava, C. N. Takacs, J. Clardy, M. A. de Pedro, M. K. Waldor, Science 2009, 325, 1552-1555.

[6] J. Sasabe, M. Suzuki, Front. Microbiol. 2018, 9: 933

[7] K. Jozwiak, W. Lough, I. Wainer in Drug Stereochemistry (Eds.: K. Jozwiak, W. Lough, I. Wainer), CRC Press, Boca Raton, 2012, pp. 1332.

[8] N. Berova, P. L. Polavarapu, K. Nakanishi, R. W. Woody in Comprehensive Chiroptical Spectroscopy, Vol. 1 and 2 (Eds.: N Berova, P. L. Polavarapu, K. Nakanishi, R. W. Woody), Wiley-VCH, 2012, pp. 1-853

[9] D. Patterson, M. Schnell, J. Doyle, Nature 2013, 497, 475-477.

[10] V. Schurig in Differentiation of Enantiomers II (Ed.: V. Schurig), Springer International Publishing, Switzerland, 2013, pp. 1-348.

[11] A. J. Hutt, B. K. Patel in Enantiospecific bioanalysis: techniques and applications. In Drug Metabolism: Towards the Next Millennium (Ed.: N J. Gooderham), IOS Press, Amsterdam, 1998, pp. 196-212.

[12] M. M. R. Fanood, N. B. Ram, C. S. Lehmann, I. Powis, M. H. M. Janssen, Nat. Commun. 2015, 6: 7511.

[13] C. Guo, R. D. Shah, R. K. Dukor, X. Cao, T. B. Freedman, L. A. Nafie, Anal. Chem. 2004, 76, 6956-6966.

[14] Y. Li, L. Wen, H. Meng, J. Lv, G. Luo, Y. Zhao, Cell Rep. Phys. Sci. 2020. DOI: $10.1016 /$ j.xcrp.2020.100100

[15] M. Pérez-Trujillo, J. C. Lindon, T. Parella, H. C. Keun, J. K. Nicholson, T. J. Athersuch, Anal. Chem. 2012, 84, 2868-2874.

[16] F. Balzano, G. Uccello-Barretta, F. Aiello in Chiral Analysis. Advances in Spectroscopy, Chromatography and Emerging Methods (Ed.: P. L. Polavarapu), Elsevier, 2018, pp. 367-427.

[17] M. Pérez-Trujillo, E. Monteagudo, T. Parella, Anal. Chem. 2013, 85 10887-10894.

[18] M. Pérez-Trujillo, A. Virgili, Tetrahedron: Asymmetry 2006, 17, 2842 2846 
[19] T. J. Wenzel, J. E. Thurston, J. Org. Chem. 2000, 65, 1243-1248.

[20] Y. Machida, M. Kagawa, H. Nishi, J. Pharm. Biomed. Anal. 2003, 30, 1929-1942.

[21] a) M. Foroozandeh, R. W. Adams, P. Kiraly, M. Nilsson, G. A. Morris, Chem. Commun. 2015, 51, 15410-15413; b) J. Aguilar, S. Faulkner, M. Nilsson, G. Morris, Angew. Chem. Int. Ed., 2010, 49, 3901-3903.

[22] D. Jeannerat, Magn. Reson. Chem. 2003, 41, 3-17.

[23] M. Pérez-Trujillo, L. Castañar, E. Monteagudo, L. T. Kuhn, P. Nolis, A. Virgili, R. T. Williamson, T. Parella, Chem. Commun. 2014, 50, 10214 10217.

[24] E. L. Ulrich; A. Hideo; J. F. Doreleijers; Y. Harano; Y. E. Ioannidis; J. Lin; M. Livny; S. Mading; D. Maziuk; Z. Miller; E. Nakatani; C. F. Schulte; D. E. Tolmie; R. K. Wenger; H. Yao; J. L. Markley; Nucleic Acids Research 2008, 36, D402-D408.

[25] M. Pérez-Trujillo, T. Parella, L. T. Kuhn, Anal. Chim. Acta 2015, 876, 63-70.

[26] T. Wang, Q. Liu, M. Wang, J. Zhou, M. Yang, G. Chen, F. Tang, E. Hatzakis, L. Zhang, Anal. Chem. 2020, 92, 3636-3642.

[27] S. K. Bharti, R. Roy, Trend. Anal. Chem. 2012, 35, 5-26.

[28] R. Altenburger, M. Scholze, W. Busch, B. I. Escher, G. Jakobs, M. Krauss, J. Krüger, P. A. Neale, S. Ait-Aissa, A. C. Almeida, T.-B. Seiler, F. Brion, K. Hilscherová, H. Hollert, J. Novák, R. Schlichting, H. Serra, Y. Shao, A. Tindall, K. E. Tollefsen, G. Umbuzeiro, T. D. Williams, A Kortenkamp, Environ. Int. 2018, 114, 95-106.
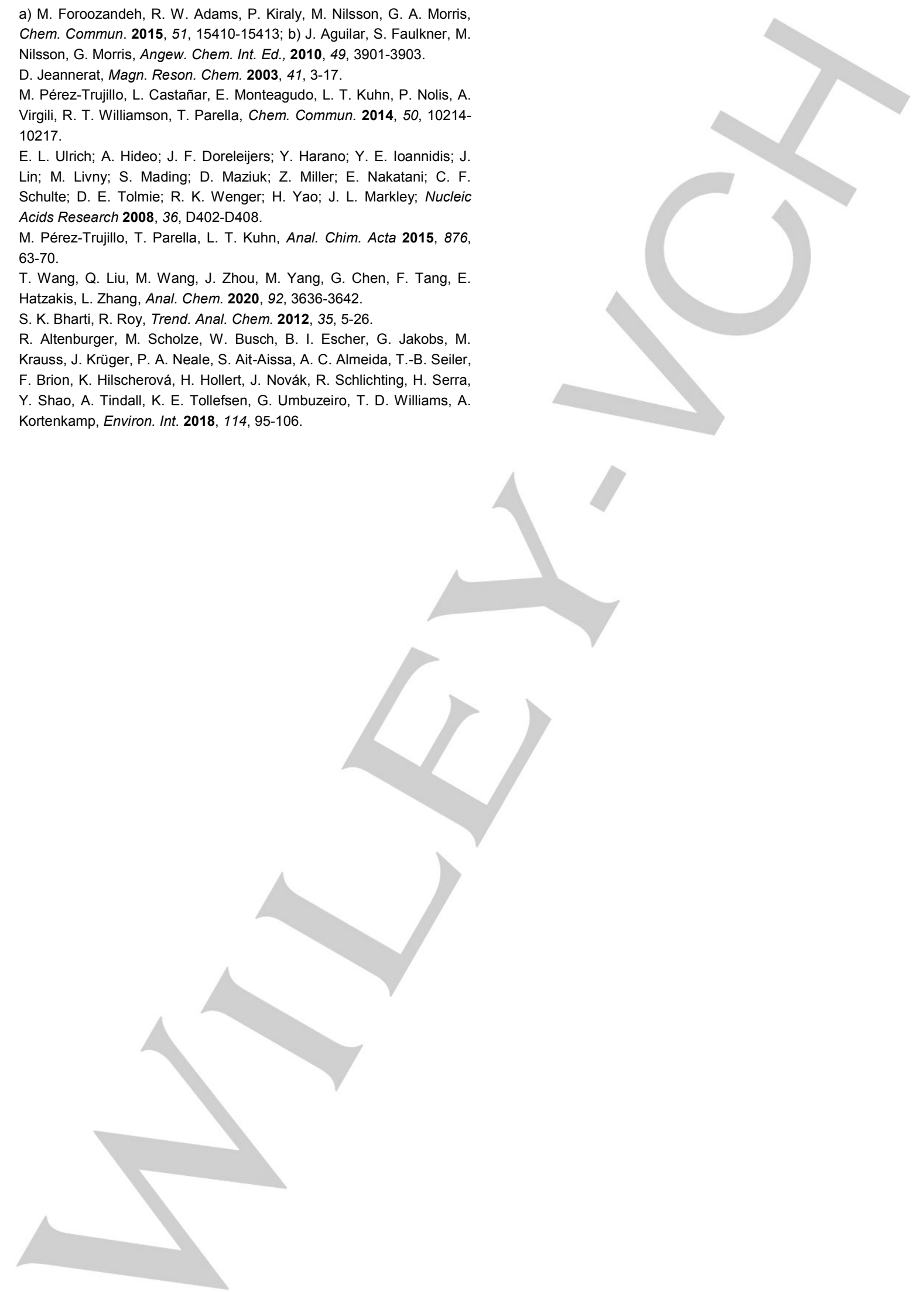


\section{Entry for the Table of Contents}
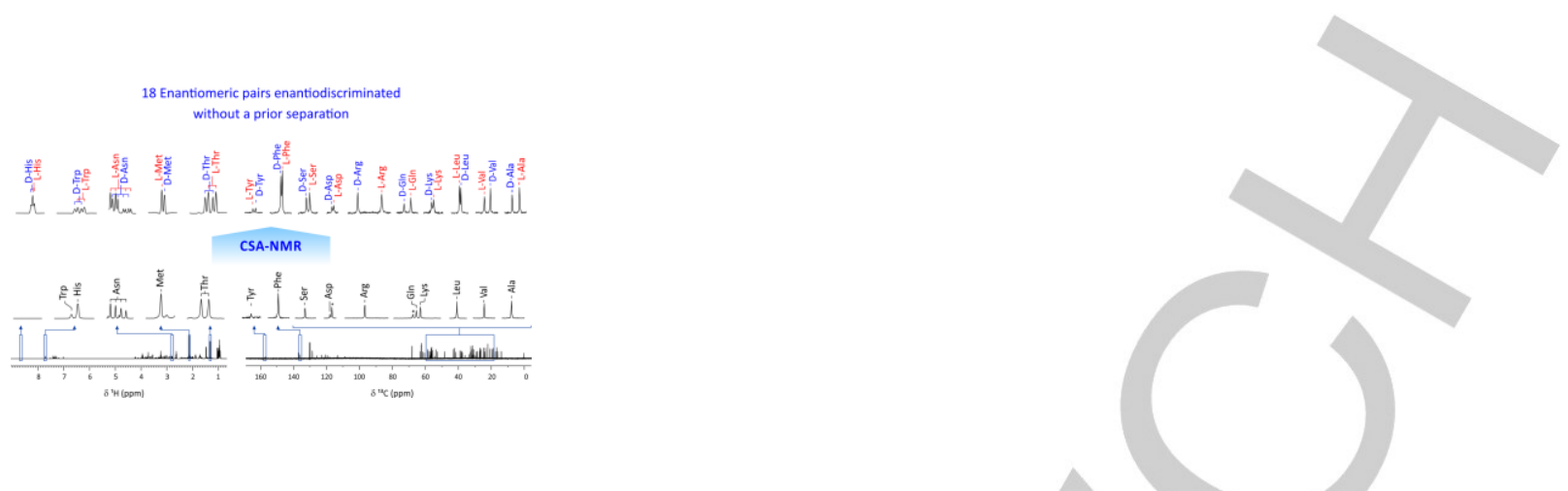

The simultaneous enantiospecific detection of multiple chiral metabolites in an aqueous mixture at physiological concentrations is reported for the first time. Eighteen essential AAs were enantiospecifically detected directly within a mixture using NMR spectroscopy and a small quantity of a chiral solvating agent. This work represents a first step towards the enantiospecific analysis of complex mixtures without previous separation of its components. 